\title{
整体叶盘通道去余量线切割加工技术研究*
}

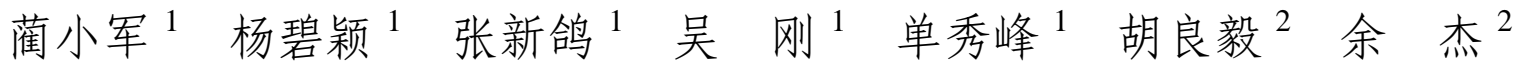

(1. 西北工业大学现代设计与集成制造技术教育部重点实验室 西安 710072;

2. 中国航发西安航空发动机有限公司 西安 710021)

\begin{abstract}
摘要: 整体叶盘的加工余量主要是在其通道开槽粗加工阶段去除的, 合理选取整体叶盘粗加工通道余量去除方法是降低通道 加工费用、实现整体叶盘加工经济性的关键。由于线切割机床加工单位成本低的原因, 采用线切割加工方法对整体叶盘通道 进行去余量加工。提出利用叶型与轮毂偏置面确定通道加工边界; 通过比较通道边界投影后的投影面积大小确定最优矢量; 然后根据最优矢量确定线切割丝与叶盘端面之间的夹角以及切割区域, 实现整体叶盘粗加工阶段去除余量最大化。该方法可 以有效地解决整体叶盘粗加工阶段经济性问题。试验表明, 线切割加工可以应用在整体叶盘通道去余量加工中, 并且与传统 数控铣削相比, 单个通道的加工费用仅为数控铁削加工费用的 $1 / 20$ 。
\end{abstract}

关键词: 整体叶盘; 线切割; 最优矢量; 通道余量

中图分类号: TP391

\section{Study on Wire Cutting Machining Techniques in Removing Allowance of Blisk Tunnel}

\author{
LIN Xiaojun $^{1} \quad$ YANG Biying $^{1} \quad$ ZHANG Xinge $^{1} \quad$ WU Gang $^{1}$ \\ SHAN Xiufeng ${ }^{1} \quad$ HU Liangyi $^{2} \quad$ YU Jie $^{2}$
}

(1. Key Laboratory of Contemporary Design and Integrated Manufacturing Technology of Ministry of Education , Northwestern Polytechnical University, Xi’an 710072;

2. AECC Xi'an Aero-engine LTD, Xi'an 710021)

\begin{abstract}
Machining allowance for a blisk is mainly removed in the rough milling, so selecting the appropriate machining method to remove the allowance of blisk tunnel in the rough milling is key to reducing the tunnel cutting costs and making the blisk machining economically. As the wire cut electrical discharge machining machine processing unit cost is low, wire cutting is used to remove the allowance of tunnel. The processing boundary of tunnel is determined by the offset surface of blade and hub of blisk.The optimal vector is determined by comparing the size of the projected area after the projection of the boundary of the tunnel. Then, the cutting area and the angle between the line cutting wire and the end face of the blisk are determined according to the optimal vector, and the cutting area is maximized. This method can effectively solve the economic problems of blisk in the rough milling. The demonstration shows that the wire cutting can be applied to remove the allowance of blisk, and compared with the traditional CNC milling, the processing cost of a single channel is only one-twentieth of the cost of CNC milling.
\end{abstract}

Key words: blisk; wire cutting; optimal vector; allowance of tunnel

\section{0 前言}

整体叶盘是现代高推重比航空发动机采用的新 结构, 与传统叶片和轮盘装配结构相比, 整体叶盘 结构布局上采用轮毂与叶片一体化的形式, 减少结 构间的连接，避免榫头气流损失、减少结构重量和

* 国家科技重大专项(2015ZX04001003)资助项目。20180405 收到初稿, 20181018 收到修改稿
零件的数量 ${ }^{[1]}$; 在气动布局上采用宽弦、弯掠叶片 和窄流道的设计, 提高气动效率, 改善气动性能, 同时使发动机结构大为简化, 提高推重比, 延长使 用寿命, 提高可靠性 ${ }^{[2]}$ 。目前, 在新型发动机设计 中普遍采用整体叶盘结构。

整体叶盘的结构形式主要有开式结构一不带外 箍整体叶盘和闭式结构一带外䈨整体叶盘两种典型 结构(图 1)。整体叶盘叶片薄、扭曲度大、叶展长、 受力易变形; 叶片通道深而窄、开敞性很差; 材料 
多为钛合金、高温合金等难加工材料; 因此, 整体 叶盘的制造技术难度较大。

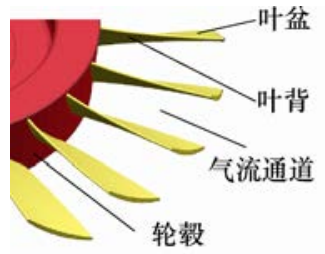

(a)开式整体叶盘

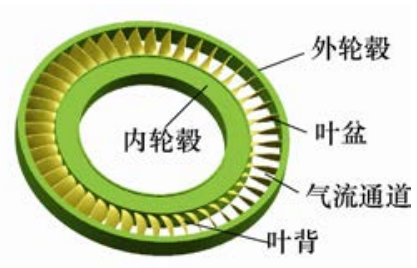

(b) 闭式整体叶盘
图 1 典型整体叶盘结构

整体叶盘加工工艺一般分为通道去余量粗加工和 型面精加工两个部分。通道开槽粗加工的材料切除量 最大, 占整体叶盘材料切除总量的 $90 \%$ 以上 ${ }^{[3]}$ 。因此, 整体叶盘通道开槽粗加工方法的优劣对其加工效率具 有重要的影响，也是实现叶盘经济性加工的关键。

目前整体叶盘通道粗加工去余量有两种常用方 法, 即数控铣削和数控电解。其中, 数控铣削技术 是整体叶盘通道加工最常用的方法, 许多学者对此 进行了研究。传统铣削方式是使用立铣刀在五坐标 数控机床上分层侧铣。单晨伟等 ${ }^{[4]}$ 针对开式整体叶 盘四坐标侧铣开槽技术进行研究, 提出一种基于特 征的侧铣开槽粗加工方法。在侧铣时刀具受径向作 用力较大, 使刀具刚性随铣削深度的增加而下降, 在径向力不断作用下, 刀具磨损、变形、振动加剧, 加工效率显著降低 ${ }^{[5-8]}$ 。王增强等 ${ }^{[9-10]}$ 基于对叶片通道 进行直纹包络面逼近来确定粗加工区域的原理对叶 盘的四坐标和五坐标的插铣方法进行研究。李活等 ${ }^{[11]}$ 提出一种基于最小二乘法原理的整体叶盘插铣开粗 算法, 可以使插铣后的叶片余量均匀分配于各叶片 型值点。董雷等 ${ }^{[12]}$ 将定轴插铣加工的理论模型概括 为通道曲面最大内接柱面优化问题并给出计算刀心 位置的迭代算法。BALAM 等 ${ }^{[13]}$ 提出了一种五坐标粗 加工刀位轨迹生成的方法。数控铣削方法存在多坐 标数控编程难, 加工材料难切削, 刀具易产生干涉, 切削力大，叶片易变形，加工成本高等问题。

在数控电解 ${ }^{[14-16]}$ 加工方面, 朱永伟等 ${ }^{[17]}$ 提出了 采用数控展成电解方法加工整体叶轮扭曲叶片。徐 庆等 ${ }^{[18]}$ 提出了电极运动轨迹优化方法。电解加工电 流要求高, 电解液和电解产物需要专门回收处理, 环境污染严重。

在线切割加工方面, 比利时鲁汶大学的 LAUWERS 等 ${ }^{[19]}$ 研究了 WC 基金属陶瓷的成分和晶 粒尺寸对线切割加工该材料可制造性的影响。HAN 等 ${ }^{[20]}$ 开发了一个 WEDM(Wire cut electrical discharge machining, WEDM)放电过程的自适应控 制模拟系统, 该系统能够产生优化参数来进行高精
度的 WEDM 加工。LIAO 等 ${ }^{[21]}$ 将神经网络引入了线 切割控制系统中, 使线切割数控更智能化和自动化。 蒲一民 ${ }^{[22]}$ 研究慢走丝线切割加工榫槽的工艺方法, 优化其加工工艺参数。赵万生等 ${ }^{[23]}$ 研制了 “轴联动 数控电火花加工机床”, 为制造闭式整体浴轮叶盘提 供了一套可行的技术解决方案。

线切割加工方法与前两种方法相比, 具有设备 低廉, 加工成本低, 经济性好的优点 ${ }^{[24]}$ 。另外, 可 以避免数控铣削过程中的刀具振动、刀具磨损、数 控编程难、由于切削力大造成的变形等问题。基于 此，提出采用线切割加工方法进行整体叶盘通道去 余量加工, 对线切割丝相对叶盘端面倾斜角度矢量 和切割范围的计算方法进行研究。

\section{1 整体叶盘通道线切割加工}

整体叶盘通道结构复杂，沿叶盘轴线看相邻叶 片互相遮挡，如图 1 所示。如果线切割丝与叶盘端 面垂直，由于遮挡原因叶盘就无法线切割加工或切 掉的余量很小。但当叶盘倾斜一定的角度后，叶片 通道会使无遮挡部分增大, 如图 1b 所示, 在这种情 况下就可以进行线切割加工整体叶盘通道, 实现去 余量。所以如何确定线切割丝相对叶盘端面倾斜角 度就非常重要。本文提出了采用投影面积法进行最 优矢量的搜索, 通过计算最优矢量, 确定线切割丝 与叶盘端面的夹角, 实现整体叶盘线切割去余量加 工。

\section{1 整体叶盘加工通道及边界}

闭式整体叶盘的气流通道由内轮晸曲面、叶盆 曲面、叶背曲面与外轮晸曲面构成如图 $1 \mathrm{~b}$ 所示。而 开式整体叶盘的气流通道比闭式整体叶盘少了外轮 觳曲面, 如图 1a 所示。这样, 在线切割加工整体叶 盘通道时, 开式叶盘可以直接切入，而闭式叶盘需 要预先打穿丝孔。

以闭式叶盘为例, 如图 2a 所示, 叶盘通道边界 由叶盆曲面 $S_{\mathrm{d}}$ 、叶背曲面 $S_{\mathrm{b}}$ 、内轮毂曲面 $S_{\mathrm{n}}$ 和外 轮毂曲面 $S_{\mathrm{w}}$ 组成。线切割加工的通道曲面边界如图 $2 \mathrm{~b}$ 所示, 由叶盆偏置曲面 $S_{\mathrm{d} z}$ 、叶背偏置曲面 $S_{\mathrm{bz}}$ 、

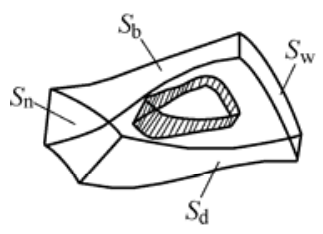

(a)

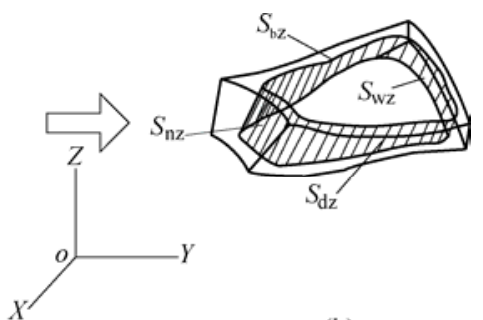

(b)
图 2 叶盘通道曲面及加工边界曲面 
内轮毂偏置曲面 $S_{\mathrm{nz}}$ 、外轮珲偏置曲面 $S_{\mathrm{wz}}$ 组成, 其 偏置量为线切割加工余量与线切割丝半径之和, 余 量大小由机床加工误差、线切割后叶片变形量和精 加工余量确定。

\section{2 搜索最优矢量}

线切割时整体叶盘通道的材料去除量大小由线 切割丝相对叶盘端面倾斜角度决定, 所以如何确定 倾斜角是去余量效率的关键。而确定倾斜角度的原 则就是整体叶盘通道余量去除最大化。在忽略高度 变化影响的情况下, 面积越大去除量体积越大。

下文以闭式叶盘为例, 搜索通道线切割最优矢 量, 最优矢量就是叶盘通道线切割的最佳角度矢量。

投影面积法就是将整体叶盘通道各偏置曲面沿 某矢量投影在以该矢量为法矢的平面上, 围成一个 封闭的区域, 比较沿不同矢量方向的投影面积大小, 其中投影面积最大时的矢量, 为最优矢量。

基于投影面积法计算最优矢量的流程如图 3 所 示, 具体步骤如下。

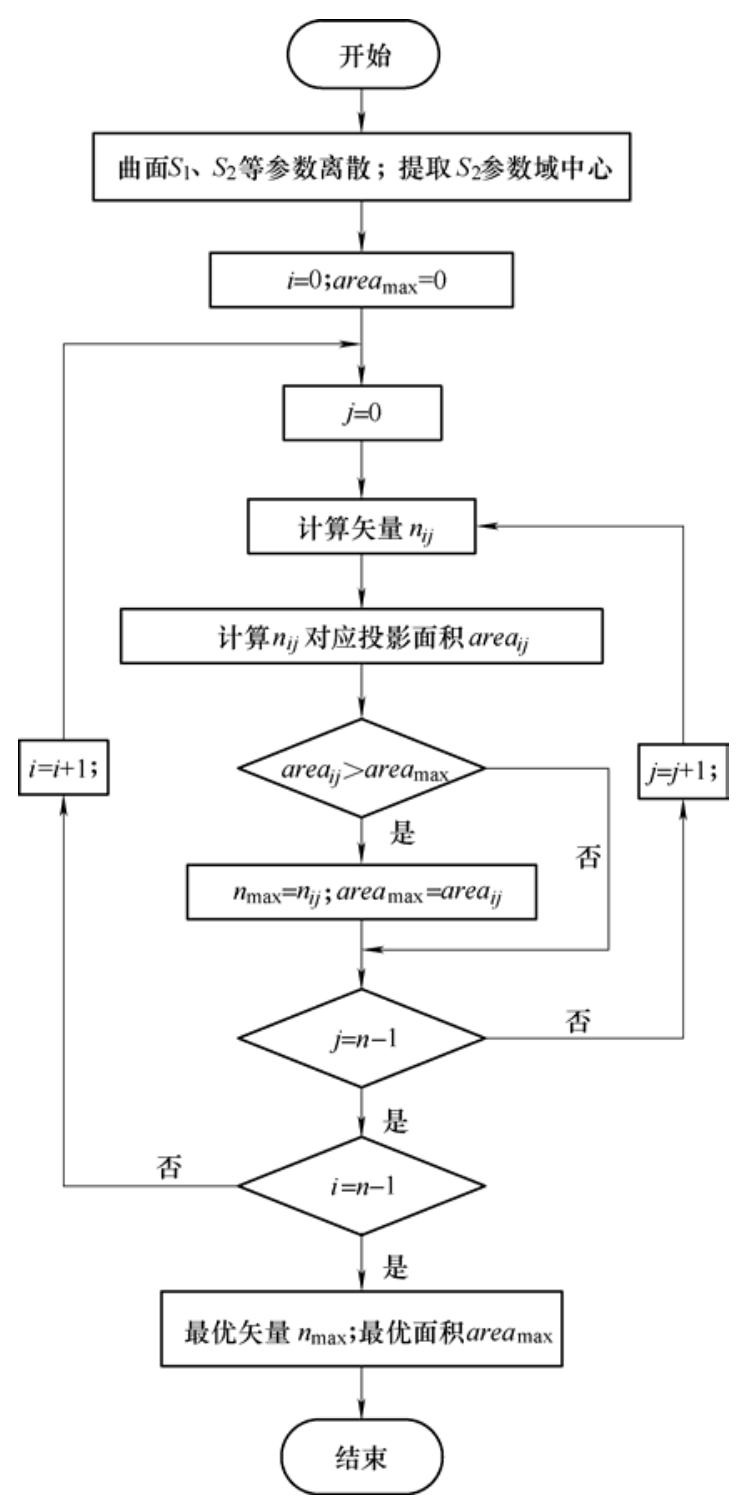

图 3 最优矢量搜索算法流程
(1) 确定通道曲面 $S_{1}$ 和 $S_{2}$ 。通道曲面 $S_{1}$ 和 $S_{2}$ 由 叶盆偏置曲面 $S_{\mathrm{d} z}$ 和叶背偏置曲面 $S_{\mathrm{bz}}$ 上下两端的母 线, 根据双线性插值来构造, 如图 4 所示, 图中省 略了外轮毂偏置面。

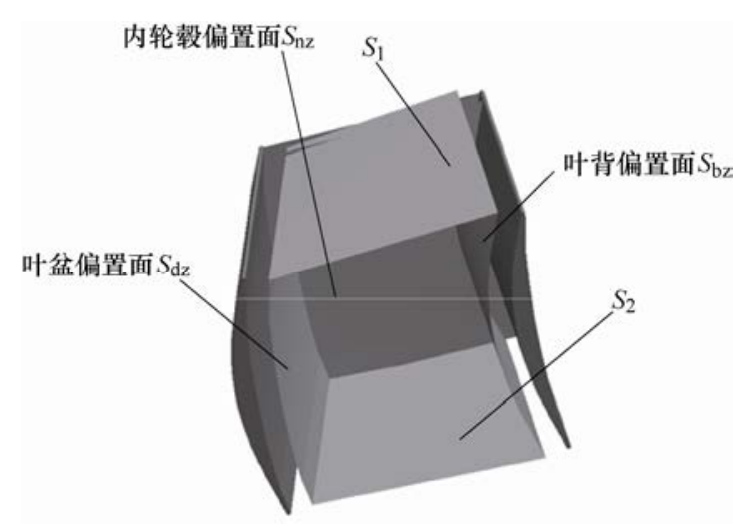

图 4 通道曲面

(2) 确定投影矢量。如图 5 所示, 首先将曲面 $S_{2}$ 等参数离散, 取参数域中心点记为 $P_{0}$ 。然后将平面 $S_{1}$ 等参数离散成 $N \times N(N=0,1, \cdots, n-1)$ 条 $U V$ 线。 将参数线 $U_{i}$ 和 $V_{j}$ 交点记为 $P_{i j}(i, j=0,1, \cdots, n-1)$ 。最后 将点 $P_{0}$ 和点 $P_{i j}$ 构造矢量 $\boldsymbol{n}_{i j}(i, j=0,1, \cdots, n-1)$, 作为投 影矢量。

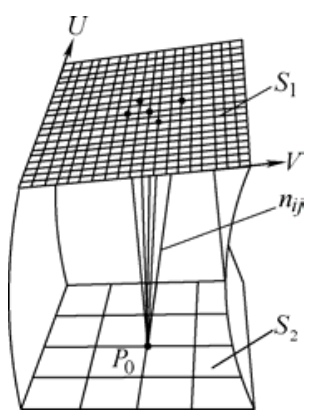

图 5 构造投影矢量

(3) 创建投影平面。如图 6 所示, 以 $\boldsymbol{n}_{i j}(i, j=0$, $1, \cdots, n-1)$ 为法矢做过点 $P_{0}$ 的平面, 记为平面 $\mathrm{F}$ 。

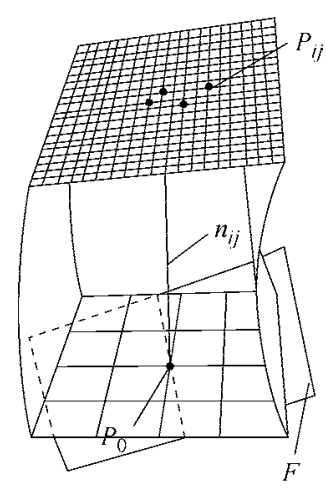

图 6 构造投影平面

(4) 确定投影边界。如图 7 所示, 将叶盆偏置 曲面 $S_{\mathrm{d} z}$ 、叶背偏置曲面 $S_{\mathrm{bz}}$ 、内轮毂偏置曲面 $S_{\mathrm{nz}}$ 以 及外轮毂偏置曲面 $S_{\mathrm{wz}}$ 按等参数离散成点。 


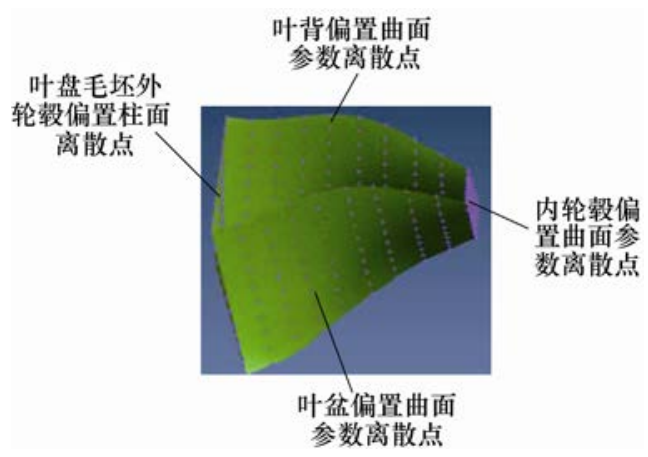

图 7 曲面离散点

将离散点集沿矢量 $\boldsymbol{n}_{i j}$ 方向投影到投影平面上, 通过点云边界提取算法 ${ }^{[25]}$ 处理求出投影边界 $H_{i j}(i, j=0,1, \cdots, n-1)$, 如图 8 所示。

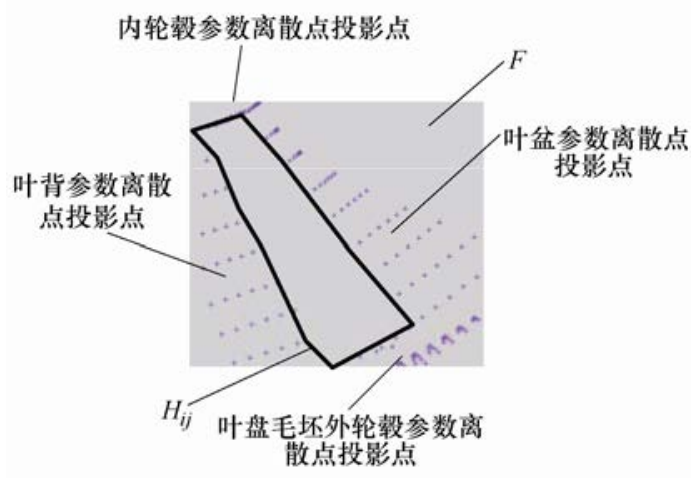

图 8 投影离散点拟合边界区域 $H_{i j}$

（5）搜索并确定最优矢量。依次计算所有 $\boldsymbol{n}_{i j}$ 情 况下的投影面积，其中最大值所对应的投影矢量记 为最优矢量 $\boldsymbol{n}_{0}$ 。最后计算出线切割丝与叶盘端面的 夹角。

\section{3 实例}

以某型开式整体叶盘为例进行整体叶盘通道线 切割去余量加工试验。采用普通线切割机床, 即这 类机床只有工作台 $X 、 Y$ 两个数控轴, 线切割丝在 切割时始终处于垂直于工作台台面状态, 为了实现 线切割丝相对叶盘端面倾斜一定角度(不是垂直), 只能通过工装使叶盘相对机床工作台面倾斜一定角 度。

试验采用 DK7740 E 电火花数控线切割机床, 叶盘材料为 TC11 钛合金, 直径 $360 \mathrm{~mm}$, 叶盘毛坏 最终零件模型是沿轮盘周向均匀分布 39 个叶片。

根据前文中最优矢量搜索算法, 求出最优矢量 为 $\boldsymbol{n}_{0}=[0.681,0.197,-0.705]$, 这样叶盘线切割倾斜装 夹的角度为绕 $Y$ 轴 $44^{\circ}$, 绕 $X$ 轴 $-11.4^{\circ}$ 。图 9a 是叶 盘无倾斜, 相邻叶片把通道完全遮挡。图 $9 \mathrm{~b}$ 是叶盘 绕 $Y$ 轴旋转后通道情况。图 9c 是叶盘绕 $Y$ 轴旋转基 础上再绕 $X$ 轴旋转后的情况。最终加工就按图 9c 进行加工。图 10 是叶盘通道线切割后所留的余量。

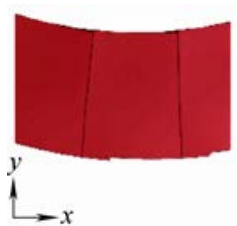

(a)

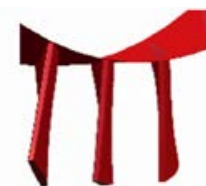

(b)

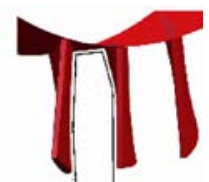

(c)
图 9 叶盘通道倾斜变化

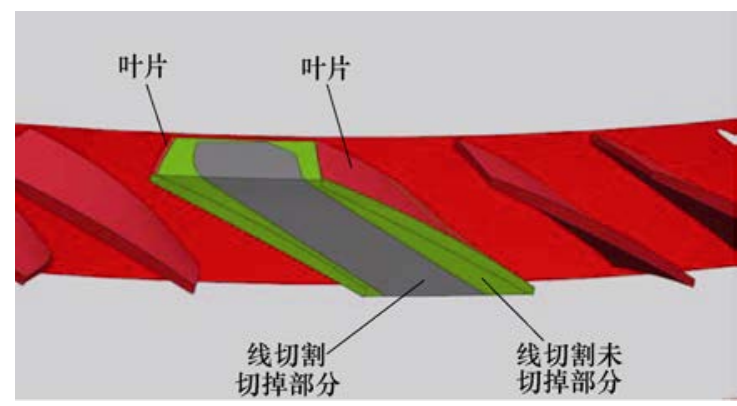

图 10 叶盘通道线切割后余量

根据前文方法得到的线切割走丝轨迹为图 11。 图 12 为整体叶盘线切割加工区去余量试验照片。图 13 为叶盘线切割加工后的通道照片，经过测量机测 量, 线切割加工面余量满足要求。

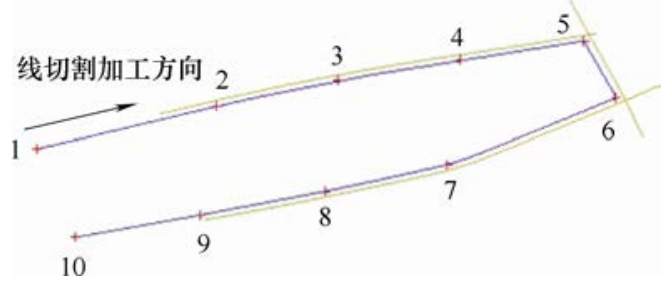

图 11 线切割加工轨迹

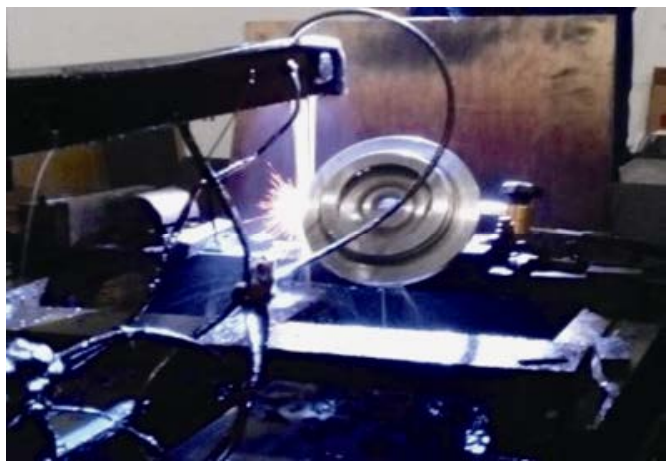

图 12 线切割加工试验

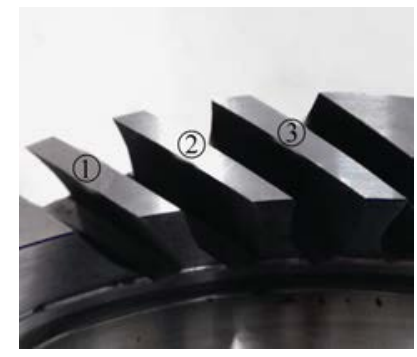

图 13 叶盘线切割加工后的通道

将叶盘单个通道去余量多轴数控铣削和线切割 进行对比，去除量、加工成本如表 1 所示。 
表 1 整体叶盘单个通道粗加工成本比较

\begin{tabular}{ccc}
\hline & 去除量占百分比 $(\%)$ & 单个通道成本/元 \\
\hline 多轴数控铣削 & 90 & 160 \\
线切割加工 & 72.2 & 8 \\
\hline
\end{tabular}

从表 1 中可以看出在粗加工阶段, 线切割加工 对单个通道的加工费用仅为数控铣削加工费用的 $1 / 20$, 这样针对通道粗加工将节省大量费用。因此, 在叶盘通道粗加工阶段采用线切割加工方法, 单位 体积去除费用低，其经济性非常显著。

\section{2 结论}

（1）提出了采用线切割方法对整体叶盘通道进 行去余量加工, 并通过试验进行了验证。试验表明: 线切割加工可以应用在整体叶盘通道去余量加工 中, 并且显著降低了加工成本。

（2）线切割丝与叶盘端面之间的夹角决定去除 余量的大小。利用叶型和轮㪍曲面偏置面来确定通 道加工边界, 采用投影面积法搜索最优矢量, 并根 据最优矢量确定线切割丝与叶盘端面夹角和切割区 域, 实现去除余量最大化。

(3) 对同一个通道进行多次切割使切除余量最 大化, 以去除余量体积最大为目标, 以及采用多轴 线切割机床(线切割丝在工作时可以两个方向倾斜 角度)进行切割技术可以进一步研究。

(4) 本文方法可以推广到线切割机床对整体叶 盘去余量加工。

\section{参 考 文 献}

[1] 任军学, 张定华, 王增强, 等. 整体叶盘数控加工技术 研究[J]. 航空学报, 2004, 25(2): 205-208.

REN Junxue, ZHANG Dinghua, WANG Zengqiang, et al. Research on the NC machining technique of blisk[J]. Acta Aeronautica et Astronautica Sinica, 2004, 25(2): 205-208.

[2] 张学超, 史耀耀, 任军学, 等. 闭式叶盘叶身加工切触 点规划算法研究与实现 $[\mathrm{J}]$. 航空精密制造技术, 2006, 42(8): 34-37.

ZHANG Xuechao, SHI Yaoyao, REN Junxue, et al. Research and implementation of programming algorithm of closed blisk's blade machining contact points[J]. Aviation Precision Manufacturing Technology, 2006, 42(8): 34-37.

[3] 任军学, 姜振南, 姚倡锋, 等. 开式整体叶盘四坐标高 效开槽插铣工艺方法 [J]. 航空学报, 2008, 29(6): 1692-1698.

REN Junxue, JIANG Zhengnan, YAO Changfeng, et al.
Process for 4-axis high efficiency slot plunge milling of open blisk[J]. Acta Aeronautica et Astronautica Sinica, 2008, 29(6): 1692-1698.

[4] 单晨伟, 任军学, 张定华, 等. 开式整体叶盘四坐标侧 铣开槽粗加工轨迹规划 [J]. 中国机械工程, 2007, 18(16): 1917-1920.

SHAN Chenwei, REN Junxue, ZHANG Dinghua, et al. 4-axis NC side milling roughing path planning for open blisks[J]. China Mechanical Engineering, 2007， 18(16): 1917-1920.

[5] 任军学, 田卫军, 田荣金鈴, 等. 开式整体叶盘通道侧铣 粗加工技术的研究[J]. 机械科学与技术, 2008, 27(10): $1220-1224$

REN Junxue, TIAN Weijun, TIAN Rongxin, et al. A study of the rough milling technique of blisk tunnel[J]. Mechanical Science and Technology for Aerospace Engineering, 2008, 27(10): 1220-1224.

[6] DE LACALLE L N L, LAMIKIZ A, SANCHEZ J A, et al. Tool path selection based on the minimum deflection cutting forces in the programming of complex surfaces milling[J]. International Journal of Machine Tools and Manufacture, 2007, 47(2): 388-400.

[7] ALTINTAS Y, ENGIN S. Generalized modeling of mechanics and dynamics of milling cutters[J]. Annals of the CIRP, 2001， 50(1): 25-30.

[8] ARMAREGO E J A, DESHPANDE N P. Computerized predictive cutting models for force in end-milling including eccentricity effects[J]. Annals of the CIRP, 1989, 38(1): 45-49.

[9] 王增强, 单晨伟, 任军学, 等. 开式整体叶盘四坐标插 铣开槽粗加工刀位轨迹规划[J]. 航空制造技术, 2007 (2): 94-97.

WANG Zengqiang, SHAN Chenwei, REN Junxue, et al. Generating 4-axis plunge roughing paths for open blisks[J]. Aeronautical Manufacturing Technology, 2007, 2: 94-97.

[10] 胡创国, 张定华, 任军学, 等. 开式整体叶盘通道插铣 粗加技术的研究 [J]. 中国机械工程, 2007, 18(2): 153-155.

HU Chuangguo, ZHANG Dinghua, REN Junxue, et al. Research on the plunge milling of blisk tunnel[J]. China Mechanical Engineering, 2007, 18(2): 153-155.

[11] 李活, 陈五一, 陈彩红. 整体叶轮插铣粗加工算法 $[\mathrm{J}]$. 计算机集成制造系统，2010，16(8)：1696-1701.

LI Tian, CHEN Wuyi, CHEN Caihong. Rough machining method for blink plunge milling[J]. Computer Integrated Manufacturing Systems, 2010, 16(8): 1696-1701.

[12] 董雷, 曹利新. 通道曲面的柱面逼近方法及其在叶轮插 铣中的应用[J]. 航空学报, 2014, 35(8): 2331-2340. 
DONG L, CAO L X. Approximation method of tunnel surfaces with general cylindrical surfaces and its application in plunge milling of impellers[J]. Acta Aeronautica et Astronautica Sinica, 2014, 35(8) : 2331-2340.

[13] BALA M, LAX P, SARMA S, et al. Generating 5 -axis NC roughing paths directly from a tessellated representation [J]. Computer - aided Design, 2000, 32(4): 261-277.

[14] RISKO D G. DAVYDOV A D. Manufacturing applications and productivity limitations of electrochemical machining[J]. Manufacturing Science and Engineering, 1993, 64: 701-711.

[15] 朱永伟, 徐家文. 复合平面摆动展成电解加工整体构件 异形面的成形分析及应用[J]. 机械工程学报, 2008, 44(12): 280-286.

ZHU Yongwei, XU Jiawen. Shaping law and process study on combined wobbling feeds for electrochemieal contour evolution machining integral component parts[J]. Chinese Journal of Mechanical Engineering, 2008, 44(12): 280-286.

[16] 朱荻, 曲宁松, RAJURKAR K P, 等. 电极平动式电解 孔加工技术研究 [J]. 机械工程学报, 2001, 37(5): 105-109.

ZHU Di, QU Ningsun, RAJURKAR K P, et al. Investigation on electrochemical machining with the orbital movement of electrode[J]. Chinese Journal of Mechanical Engineering, 2001，37(5): 105-109.

[17] 朱永伟, 徐家文, 胡平旺. 数控展成电解加工整体叶轮 的研究与应用 $[\mathrm{J}]$. 航空学报, 2001, 22(4)：376-378.

ZHU Yongwei, XU Jiawen, HU Pingwang. Study and application of NC-electrochemical contour evolution machining intergral impeller[J]. Acta Aeronautica et Astronautica Sinica, 2001, 22(4): 376-378.

[18] 徐庆, 朱荻, 徐正杨, 等. 整体叶盘通道电解加工电极 多维运动轨迹优化 [J]. 航空学报, 2011, 32(8): 1548-1554.

XU Qing, ZHU Di, XU Zhengyang, et al. Optimization of cathode multidimensional movement path in electrochemical machining of blisk tunnels[J]. Acta
Aeronautica et Astronautica Sinica, 2011, 32(8) : 1548-1554.

[19] LAUWERS B, LIU W, Eeraerts W. Influence of the composition of WC-based cermets on manufacturability by wire-EDM [J]. Journal of Manufacturing Processes, 2006, 8(2): 83-89.

[20] HAN F, KUNIEDA M, SENDAI T, et al. High precision simulation of WEDM using parametric programming [J]. CIRP Annals-Manufacturing Technology， 2002， 51(1): 165-168.

[21] LIAO Y S, YAN M T, CHANG C C. A neural network approach for the on-line estimation of workpiece height in WEDM[J]. Journal of Materials Processing Technology, 2002, 121(2): 252-258.

[22] 蒲一民. 浴轮盘榫槽线切割加工工艺研究[J]. 航天制 造技术，2016, 2: 37-40，70.

PU Yimin. Research on WEDM process of turbine groove[J]. Manufacturing Processes Research, 2016, 2: $37-40,70$

[23] 赵万生, 吴湘, 詹涵菁, 等. 带冠整体式浴轮盘的 CAD/CAM[J]. 推进技术，2003，24(4): 380-383.

ZHAO Wansheng, WU Xiang, ZHAN Hanjing, et al. CAD CAM of rimed blisks[J]. Journal of Propulsion Technology, 2003, 24(4): 380-383.

[24] 朱宁, 叶军, 韩福柱, 等. 电火花线切割加工技术及其 发展动向 $[\mathrm{J}]$. 电加工与模具, 2010(S1): 53-59.

ZHU Ning, YE Jun, HAN Fuzhu., et al. Development trends of WEDM technology[J]. Electromachining \&Mould, 2010(S1): 53-59.

[25] 孙殿柱, 朱昌志, 李延瑞. 散乱点云边界特征快速提取 算法[J]. 山东大学学报, 2009, 39(1): 84-86.

SUN Dianzhu, ZHU Changzhi, LI Yanrui. An improved extraction of boundary characteristic from scattered data[J]. Journal of Shandong University, 2009, 39(1): 84-86.

作者简介: 萄小军(通信作者), 男, 1968 年出生, 博士, 高级工程师。 主要研究方向为精密测量、逆向造型、CAD/CAM。

E-mail: linxj@nwpu.edu.cn

杨碧颖, 女, 1995 年出生。主要研究方向为 CAD/CAM。

E-mail: yby950601@163.com 